\title{
Prime power divisors of binomial coefficients: Reprise
}

\author{
By J.W. Sander at Hannover
}

In [1], we proved an old conjecture of P. Erdös by showing that, given a positive integer $j$, for each sufficiently large $n$, there is a prime $p$ such that

$$
p^{j} \mid\left(\begin{array}{c}
2 n \\
n
\end{array}\right)
$$

As K. Ramachandra recently pointed out to me, Erdös once asked him in a letter if the following holds.

Proposition. Let $j$ be a positive integer. For sufficiently large $n$, there is a prime $p$ such that

$$
p^{j} \|\left(\begin{array}{c}
2 n \\
n
\end{array}\right)
$$

i.e. $p^{j}$ is the highest power of $p$ dividing $\left(\begin{array}{c}2 n \\ n\end{array}\right)$.

This can be dealt with by the method introduced in section 5 of [1]. In particular, we consider

$$
K_{j}(n):=\operatorname{card}\left\{n^{\frac{1}{j+1}}<p<\left(\frac{3}{2} n\right)^{\frac{1}{j+1}}:\left\{\frac{n}{p^{i}}\right\}>\frac{2}{3}(2 \leqq i<j),\left\{\frac{n}{p}\right\}<\frac{1}{3}\right\},
$$

where $\{x\}$ denotes the fractional part of the real number $x$. By Theorem 3 of $[1], K_{j}(n)>0$ for sufficiently large $n$. Hence

$$
n=n_{j} p^{j}+\cdots+n_{1} p+n_{0}
$$

for some prime $p$ with $\frac{1}{2} p<n_{i}<p(1 \leqq i \leqq j)$ and $0 \leqq n_{0}<\frac{1}{3} p$. By Kummer's lemma ([1], Lemma 9), this implies 


$$
p^{j} \|\left(\begin{array}{c}
2 n \\
n
\end{array}\right)
$$

In fact, we can show much more by applying the following lemma which is a corollary to a recent upper bound for exponential sums of the type

$$
\sum_{p \leqq P} e\left(x\left(\frac{h_{1}}{p^{j_{1}}}+\cdots+\frac{h_{r}}{p^{j_{r}}}\right)\right)
$$

with $e(x):=e^{2 \pi i x}$ for real $x$.

Lemma (= Proposition 2 in [2]). For $0<\sigma_{i} \leqq 1(1 \leqq i \leqq j)$,

$$
\exp \left(c_{1} j(\log 2 j)^{3}\right) \leqq P \leqq x^{1 / j},
$$

and $0<\varepsilon \leqq 1 / 12$, we have

$$
\begin{aligned}
& \left|\operatorname{card}\left\{p \leqq P:\left\{\frac{x}{p^{i}}\right\}<\sigma_{i}(1 \leqq i \leqq j)\right\}-\sigma_{1} \ldots \sigma_{j} \pi(P)\right| \\
& \leqq c_{2}^{j}\left(P^{1-c_{3} \varepsilon v_{6}(j)(\log P / \log x)^{2}}+P^{\frac{j+2}{2}+\varepsilon} x^{-\frac{1}{2}}\right)(\log x)^{4 j}
\end{aligned}
$$

where $c_{2}$ and $c_{3}$ are some positive absolute constants, and

$$
v_{i}(j):=\frac{1}{j^{i}(\log 2 j)^{2}} .
$$

Theorem. For sufficiently large $n$, there are $s=s(n)$ primes $p_{1}, \ldots, p_{s}$ with

$$
s(n) \gg\left(\frac{\log n}{(\log \log n)^{3}}\right)^{\frac{1}{10}}
$$

such that

$$
p_{j}^{j} \|\left(\begin{array}{c}
2 n \\
n
\end{array}\right) \quad(1 \leqq j \leqq s(n)) .
$$

Proof. We follow the proof of Proposition 3 in [2]. Let $j>C_{0}$,

$$
n \geqq N_{0}:=C^{j^{10}(\log j)^{3}},
$$

where $C_{0}$ and $C$ are some absolute positive constants. Applying the lemma for $x:=n \geqq N_{0}$, $\varepsilon:=1 / 12$, and $P:=n^{1 /(j+1)}$ resp. $P:=(3 n / 2)^{1 /(j+1)}$, we obtain

$$
\left|K_{j}(n)-\left(\frac{1}{3}\right)^{j}\left(\pi\left(\left(\frac{3}{2} n\right)^{\frac{1}{j+1}}\right)-\pi\left(n^{\frac{1}{j+1}}\right)\right)\right| \leqq c_{4}^{j} n^{\frac{1}{j+1}-c_{5} v_{9}(j)}(\log n)^{4 j},
$$

where $c_{4}$ and $c_{5}$ are some absolute positive constants 
Clearly,

$$
\pi\left(\left(\frac{3}{2} n\right)^{\frac{1}{j+1}}\right)-\pi\left(n^{\frac{1}{j+1}}\right)>c_{6} \frac{n^{\frac{1}{j+1}}}{\log n}
$$

for some absolute positive constant $c_{6}$. Consequently,

$$
K_{j}(n) \geqq c_{7}^{j} \frac{n^{1 /(j+1)}}{\log n}-c_{4}^{j} n^{\frac{1}{j+1}-c_{5} v_{9}(j)}(\log n)^{4 j}
$$

for yet another positive absolute constant $c_{\eta}$.

As in the proof of Proposition 3 in [2], we conclude that $K_{j}(n)>0$. Hence, by the same argument as the one leading to (1),

$$
p_{j}^{j} \|\left(\begin{array}{c}
2 n \\
n
\end{array}\right)
$$

for some prime $p_{j}$.

In order to prove the theorem, we may assume without loss of generality that

$$
n>C^{\left(C_{0}+1\right)^{10}\left(\log \left(C_{0}+1\right)\right)^{3}} .
$$

By the above reasoning, (2) holds for any $j>C_{0}$ satisfying

$$
C^{j^{10}(\log j)^{3}} \leqq n .
$$

In other words, (2) holds for all $j$ with

$$
C_{0}<j \ll\left(\frac{\log n}{(\log j)^{3}}\right)^{\frac{1}{10}}
$$

Sufficient for this is

$$
C_{0}<j \ll\left(\frac{\log n}{(\log \log n)^{3}}\right)^{\frac{1}{10}}
$$

For $j \leqq C_{0},(2)$ is rather easy to verify if $n$ is large enough. This completes the proof of the theorem.

Remark. We end this article by pointing out that the results obtained in the Proposition and the Theorem can be extended to binomial coefficients

$$
\left(\begin{array}{l}
m \\
k
\end{array}\right)
$$

by the above methods, if $m$ is sufficiently large and $|m-k|$ is "small" in comparison with $m$ (see [1]). 


\section{References}

[1] J.W. Sander, Prime power divisors of binomial coefficients, J. reine angew. Math. 430 (1992), 1-20. [2] J.W. Sander, On the order of prime powers dividing $\left(\begin{array}{c}2 n \\ n\end{array}\right)$, to appear.

Institut für Mathematik, Universität Hannover, Welfengarten 1, D-W-3000 Hannover 1

Eingegangen 19. August 1992 